\title{
Características epidemiológicas de los pacientes atendidos por COVID-19 en el Servicio de Emergencia del Hospital Militar Central Luis Arias Schreiber
}

\author{
Katia Michelle Pezo Dianderas*1, b; Diego Rolando Chávez Fernández 2, b; Raúl Ernesto Porras Serna 3, a
}

\section{RESUMEN}

Objetivo: Determinar las características epidemiológicas de los pacientes atendidos por COVID-19 en el Servicio de Emergencia del Hospital Militar Central Luis Arias Schreiber en el periodo julio a octubre del 2020.

Materiales y métodos: Se realizó un estudio observacional, transversal y descriptivo. Fueron evaluados 89 pacientes que tenían un resultado positivo en una prueba molecular y/o rápida para SARS-CoV-2. La información se recogió del reporte diario de los pacientes hospitalizados en el Servicio de Emergencia del hospital en el periodo de julio a octubre de 2020. Los resultados fueron procesados con el paquete estadístico SPSS V 25.0.

Resultados: El sexo masculino fue el más afectado por la COVID-19 (84,27 \%). El rango de edad con mayor número de casos fue de 50 a 59 años (34,83\%). El porcentaje más alto de muertes se encontró en el grupo de los mayores de 70 años; y el 71,43 \% de los fallecidos fueron mujeres. Las comorbilidades se reportaron a partir de los 40 años, y el $67,41 \%$ de pacientes presentó al menos una de ellas. La obesidad fue la comorbilidad más frecuente, seguida por la hipertensión arterial y la diabetes mellitus tipo 2. El 67,41 \% de pacientes usaron ventilación no invasiva y la mayoría fueron hospitalizados. El 21,34 \% de pacientes requirió ventilación mecánica y el 68,42 \% fueron internados en la unidad de cuidados intensivos.

Conclusiones: Las características de mayor prevalencia en los pacientes con COVID-19 son el sexo masculino, la edad entre 50 y 59 años y la obesidad. Asimismo, el empleo de la ventilación no invasiva tuvo mejor pronóstico que el uso de la ventilación invasiva.

Palabras clave: COVID-19; Coronavirus; SARS-CoV-2; Pandemia; Epidemiología (Fuente: DeCS BIREME).

\section{Epidemiological characteristics of COVID-19 patients treated at the Emergency Department of the Hospital Militar Central Luis Arias Schreiber}

\author{
ABSTRACT
}

Objective: To determine the epidemiological characteristics of COVID-19 patients treated at the Emergency Department of the Hospital Militar Central Luis Arias Schreiber from July to October 2020.

Materials and methods: A descriptive, observational and cross-sectional study was carried out with 89 patients who tested positive for SARS-CoV-2 on molecular and/or rapid tests. The information was collected from the daily progress report of the patients treated at the hospital's Emergency Department from July to October 2020. The results were processed using the IBM SPSS Statistics V25.0.

Results: Males were more likely to test positive for COVID-19 (84.27\%). Most cases occurred in patients aged between 50 and $59(34.83 \%)$. The highest percentage of deaths was found in patients older than 70 years, out of whom $71.43 \%$ were females. Comorbidities were reported in patients aged 40 and older, out of whom $67.41 \%$ had at least one of them. Obesity was the most frequent comorbidity, followed by hypertension and type 2 diabetes mellitus. Sixty-seven point four one percent $(67.41 \%)$ of the patients required non-invasive ventilation and most of them were hospitalized. Twenty-one point three four percent (21.34\%) of the patients required mechanic ventilation and $68.42 \%$ were admitted to the intensive care unit.

Conclusions: The most common characteristics of COVID-19 patients are male sex, ages between 50 and 59 years old, and obesity. Additionally, using non-invasive ventilation had a better prognosis than using mechanical ventilation.

Keywords: Coronavirus infections; Coronavirus; SARS-CoV-2; Pandemics; Epidemiology (Source: MeSH NLM).

1 Universidad Andina del Cusco, Escuela Profesional de Medicina Humana. Cusco, Perú.

2 Universidad de San Martín de Porres, Facultad de Medicina Humana. Lima, Perú.

3 Universidad Ricardo Palma, Facultad de Medicina Humana. Lima, Perú.

a Médico Especialista en Medicina de Emergencias y Desastres.

b Estudiante de Medicina Humana.

* Autor corresponsal. 


\section{INTRODUCCIÓN}

Los coronavirus (CoVs) son patógenos importantes para los seres humanos. Dentro de estos virus destacan los SARS-CoV que, hasta este momento, se han clasificado en SARS-CoV y SARS-CoV-2. Este último causa la COVID-19, enfermedad que puede complicarse y ocasionar una neumonía severa, el síndrome de dificultad respiratoria aguda, un shock séptico y edema pulmonar, cuadros que podrían tener un desenlace fatal (1).

Valdivia et al. refieren que en 2002, en Guangdong, China, se reportó el primer brote por SARS-CoV que afectó a 8422 personas, de las cuales fallecieron $916(10,8 \%)^{(2)}$.

Hasta el 19 de noviembre del 2020, el John Hopkins University School of Medicine registra 56754669 casos confirmados que incluyen 1357641 muertes por COVID-19 en todo el mundo ${ }^{(3)}$.

En el informe N. 32 sobre el COVID-19, la OPS refiere que, hasta el 2 de noviembre del 2020, en la región de las Américas se registraron 20722940 casos confirmados y 642 995 muertes por COVID-19 ${ }^{(4)}$.

En el Perú, según el Ministerio de Salud (Minsa), hasta el 31 de octubre del presente año se reportaron 904911 casos positivos sintomáticos y 34529 defunciones confirmadas por COVID-19 (3,8 \%). A su vez, la tasa de fallecidos en los hospitales Policía Nacional del Perú y de las Fuerzas Armadas (PNP/ FF. AA.) era el 2,6\% del total de fallecidos en el país ${ }^{(5)}$.

En la actualidad, las enfermedades crónicas no transmisibles (ECNT) son uno de los mayores retos para los sistemas de salud en todo el mundo. Entre ellas están la diabetes mellitus (DM), la hipertensión arterial (HTA), el sobrepeso, la obesidad, las dislipidemias, las nefropatías, las cardiopatías y las neoplasias, entre otras. Se ha observado que la mortalidad en el SARS-CoV-2 podría ser mayor en aquellos pacientes que tienen alguna enfermedad crónica no transmisible, sobre todo en los mayores de 60 años quienes, por la edad, ya poseen el riesgo para contraer esta infección y tienen un riesgo diez veces más alto de fallecer ${ }^{(6)}$.

Diversos estudios sobre el COVID-19 han determinado cuál es el sexo más afectado, la edad de presentación más frecuente, las comorbilidades más frecuentes en los pacientes, entre otras características. Es por ello que el objetivo de esta investigación es determinar las características epidemiológicas de los pacientes con COVID-19 atendidos en el Servicio de Emergencia del
Hospital Militar Central en el periodo de julio a octubre de 2020, para así poder tener una posible referencia para futuras atenciones.

\section{MATERIALES Y MÉTODOS}

\section{Diseño y población de estudio}

El presente estudio fue descriptivo y estuvo basado en un análisis de series de tiempo correspondiente al periodo de julio a octubre del 2020 en el Servicio de Emergencia del Hospital Militar Central Luis Arias Schreiber. Se incluyó a 89 pacientes atendidos en el Servicio de Emergencia con resultado positivo a infección por SARS-CoV-2 (mediante prueba rápida o prueba molecular). El muestreo fue de tipo aleatorio simple.

\section{Variables y mediciones}

Se usó el reporte diario de pacientes con diagnóstico de COVID-19 proporcionado por el jefe del Servicio de Emergencia del Hospital Militar Central Luis Arias Schreiber.

\section{Análisis estadístico}

El registro de los datos consignados en el Servicio de Emergencia fueron procesados utilizando el paquete estadístico SPSS V 25.0.

\section{Consideraciones éticas}

Se obtuvo el permiso del Comité de Ética del Hospital Militar Central Luis Arias Schreiber y se guardó confidencialidad de los nombres de los pacientes.

\section{RESULTADOS}

De los 89 pacientes estudiados, el grupo etario de 50 a 59 años tuvo el porcentaje más alto de infección con 31 casos $(34,83 \%)$. El promedio de edad fue de 59,01 años (valor mínimo 29 años y valor máximo 87). Respecto al sexo, 75 pacientes fueron hombres $(84,27 \%)$ y 14 , mujeres $(15,73 \%)$. En el sexo femenino el grupo más numeroso estaba entre los 70 a 79 años (6 casos), además, no se reportaron mujeres menores de 50 años.

Las comorbilidades se reportaron a partir de los 40 años. De ellas, la obesidad fue la más frecuente, seguida por la HTA $y$, en tercer lugar, la DM2.

El $67,41 \%$ de los pacientes presentó una comorbilidad; el $19,10 \%$, tenía dos , y los pacientes con 3 comorbilidades fueron el 5,62 \%; mientras que los pacientes que no tuvieron ninguna comorbilidad fueron el 32,58 \% (Tabla 1). En el sexo femenino se observa que la comorbilidad más frecuente fue la DM2, con 6 casos $(35,29 \%)$. 
Características epidemiológicas de los pacientes atendidos por COVID-19 en el

Servicio de Emergencia del Hospital Militar Central Luis Arias Schreiber

Tabla 1. Número de comorbilidades y su predominancia en pacientes con COVID-19

\begin{tabular}{|c|c|c|c|c|c|c|c|c|}
\hline & \multicolumn{8}{|c|}{ Número de comorbilidades } \\
\hline & \multicolumn{2}{|c|}{$\begin{array}{c}\text { Ninguna } \\
\text { comorbilidad } \\
\end{array}$} & \multicolumn{2}{|c|}{$\begin{array}{c}1 \\
\text { comorbilidad } \\
\end{array}$} & \multicolumn{2}{|c|}{$\begin{array}{c}2 \\
\text { comorbilidades }\end{array}$} & \multicolumn{2}{|c|}{$\begin{array}{c}30 \text { más } \\
\text { comorbilidades }\end{array}$} \\
\hline & $\mathbf{N}$ & $\%$ & $\mathbf{N}$ & $\%$ & $\mathbf{N}$ & $\%$ & $\mathbf{N}$ & $\%$ \\
\hline Sin antecedentes & 29 & 100,00 & & & & & & \\
\hline Sobrepeso & & & & & 1 & 100,00 & & \\
\hline Obesidad & & & 19 & 73,08 & 3 & 11,54 & 4 & 15,38 \\
\hline Dislipidemia & & & & & 1 & 100,00 & & \\
\hline Hipertensión arterial & & & 4 & 21,05 & 11 & 57,90 & 4 & 21,05 \\
\hline Diabetes mellitus 2 & & & 7 & 41,18 & 7 & 41,18 & 3 & 17,64 \\
\hline Asma & & & 1 & 100,00 & & & & \\
\hline Otras enfermedades cardiacas & & & 1 & 25,00 & 1 & 25,00 & 2 & 50,00 \\
\hline Enfermedades oncológicas & & & 2 & 33,33 & 3 & 50,00 & 1 & 16,67 \\
\hline Otras enfermedades & & & 4 & 33,33 & 7 & 58,33 & 1 & 8,34 \\
\hline
\end{tabular}

De los 89 pacientes atendidos por COVID-19 en el Servicio de Emergencia, 44 fueron hospitalizados (49,44\%), 23 fallecieron (25,84\%), 19 pasaron a UCI $(21,35 \%)$ y 3 salieron de alta $(3,37 \%)$.

En la tabla 2 se observa que fueron hospitalizados 40 hombres $(53,33 \%)$ y 4 mujeres $(28,57 \%)$. Con respecto a los pacientes fallecidos, 10 fueron del sexo femenino
(71,43\%) y 13 , del sexo masculino $(17,34 \%)$. Los pacientes de todos los grupos etarios fueron hospitalizados, excepto los de 70 a 79 años. El grupo con mayor número de ingresos a la UCl fue el de 50 a 59 años. El mayor porcentaje de fallecidos estuvo en los pacientes de 70 a 79 años (n: 9; $81,81 \%)$, seguido por el grupo de mayores a 80 años (n: $3 ; 42,86 \%$ ) y los pacientes de 50 a 59 años (n: 6; 19,35\%).

Tabla 2. Asociación entre el sexo y el destino final de los pacientes atendidos por COVID-19 en el Servicio de Emergencia en el HMC

\begin{tabular}{clrrrr} 
& & \multicolumn{5}{c}{ Sexo } \\
\cline { 3 - 6 } & & \multicolumn{1}{c}{ Masculino } & Femenino \\
\cline { 3 - 6 } & & $\mathrm{N}$ & $\%$ & $\mathrm{~N}$ & $\%$ \\
\hline Destino & Alta & 3 & 4,00 & & \\
final & Hospitalización & 40 & 53,33 & 4 & 28,57 \\
& UCl & 19 & 25,33 & & \\
& Fallecido & 13 & 17,34 & 10 & 71,43 \\
& Total & 75 & 100,00 & 14 & 100,00
\end{tabular}

La mayoría de pacientes que requirieron ventilación mecánica no invasiva fueron hospitalizados (n: 29; 48,33 \%) y 14 pacientes $(23,33 \%)$ requirieron ser internados en $\mathrm{UCl}$. De los fallecidos, 15 (25,00\%) precisaron ventilación no invasiva (Tabla 3).

Tabla 3. Asociación entre el uso de ventilación no invasiva y el destino final de los pacientes atendidos por COVID-19 en el Servicio de Emergencia en el HMC

\begin{tabular}{|c|c|c|c|c|c|c|c|c|c|c|c|}
\hline & & \multicolumn{10}{|c|}{ Destino final } \\
\hline & & \multicolumn{2}{|c|}{ Alta } & \multicolumn{2}{|c|}{ Hospitalización } & \multicolumn{2}{|c|}{$\mathrm{UCl}$} & \multicolumn{2}{|c|}{ Fallecido } & \multicolumn{2}{|c|}{ Total } \\
\hline & & N & $\%$ & $\mathrm{~N}$ & $\%$ & $\mathbf{N}$ & $\%$ & $\mathrm{~N}$ & $\%$ & $\mathrm{~N}$ & $\%$ \\
\hline Ventilación no invasiva & Sí & 2 & 3,34 & 29 & 48,33 & 14 & 23,33 & 15 & 25,00 & 60 & 67,41 \\
\hline & No & 1 & 3,45 & 15 & 51,72 & 5 & 17,24 & 8 & 27,59 & 29 & 32,58 \\
\hline
\end{tabular}


El 68,42 \% de los pacientes que precisaron de ventilación mecánica fueron derivados a $\mathrm{UCl}(\mathrm{n}$ : 13;), mientras que la mayoría de los que no lo requirieron fueron hospitalizados ( $\mathrm{n}: 42 ; 60,00 \%$ ). De los pacientes fallecidos solo $4(17,39 \%)$ necesitaron ventilación mecánica, mientras que 19 no lo requirieron $(82,61 \%)$. Ninguna paciente mujer usó ventilación mecánica (Tabla 4).

Tabla 4. Asociación entre el uso de ventilación mecánica y el destino final de los pacientes atendidos por COVID-19 en el Servicio de Emergencia en el HMC

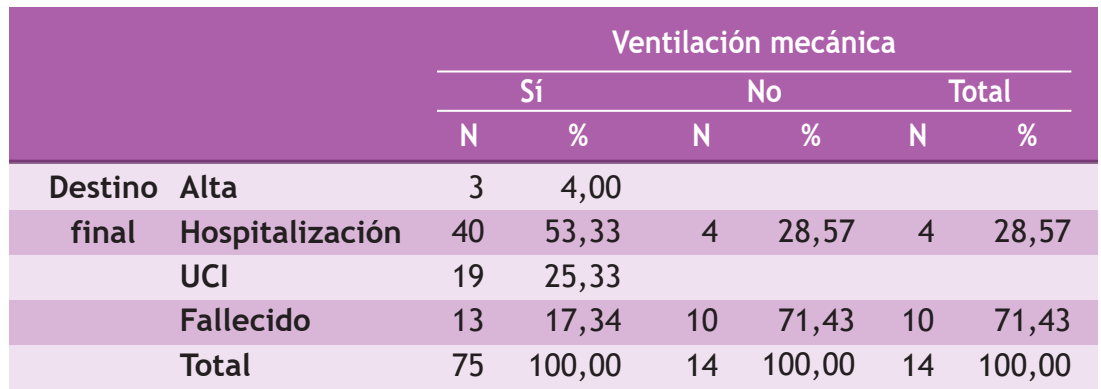

Se observa que 11 pacientes $(12,36 \%)$ usaron ventilación mecánica y no invasiva en su estadía en emergencia (Tabla 5). El 55,05 \% (9 pacientes) usó ventilación no invasiva pero no requirió ventilación mecánica; y 21 pacientes no emplearon ni ventilación no invasiva ni ventilación mecánica $(23,59 \%)$.

Tabla 5. Asociación entre el uso de ventilación no invasiva y de ventilación mecánica de los pacientes atendidos por COVID-19 en el Servicio de Emergencia en el HMC

\begin{tabular}{lrrrrr} 
& & \multicolumn{4}{c}{ Ventilación mecánica } \\
\cline { 3 - 6 } & & \multicolumn{3}{c}{ Sí } & \multicolumn{2}{c}{ No } \\
\cline { 2 - 6 } & N & $\%$ & N & $\%$ \\
\hline Ventilación no invasiva & Sí & 11 & 57,90 & 49 & 70,00 \\
& No & 8 & 42,10 & 21 & 30,00
\end{tabular}

\section{DISCUSIÓN}

El COVID-19 es una nueva enfermedad causada por el SARS-CoV-2, por ende, se requieren estudios para comprender este nuevo virus y sus efectos.

En la gran mayoría de estudios realizados en pacientes con COVID-19, se encontró que los hombres constituyen el mayor porcentaje de pacientes ${ }^{(7-11)}$, mientras que solo en tres predomina el sexo femenino ${ }^{(13,16,19)}$. El presente trabajo coincide con estas investigaciones, ya que incluyó a 75 hombres $(84,27 \%)$ y 14 mujeres $(15,73 \%)$

Según Villagran $\mathrm{K}$ et al., en ocho investigaciones sobre pacientes con COVID-19, el mayor rango de edad informado fue de los 40 a 70 años $(7,10,12,18)$. Estos resultados coinciden con nuestro reporte, ya que el mayor número de pacientes está en el grupo etario de 50 a 59 (n: $31 ; 34,83 \%$ ), seguido por el de 60 a 69 años (n: 20; 22,47\%), y en tercer lugar, los enfermos de 40 a 49 años (n: 15; 16,85\%). El promedio de edad fue de 59,01 años, con un valor mínimo de 29 años y un valor máximo 87 años.
En nuestro estudio, los pacientes hospitalizados no requirieron ser transferidos a la UCI (n: 44 y 49,44\%), como sí ocurrió en los reportes de Zhao J et al. y Llaro M et al. ${ }^{(11,18)}$.

El mayor número de los pacientes hombres tenía una edad entre 50 y 59 años y el $53,33 \%$ de ellos fue hospitalizado. Por otro lado, en el grupo femenino predominan las mujeres de 70 a 79 años $(54,55 \%)$, seguidas por las mayores de 80 años (42,86\%). Es importante mencionar que el 71,43\% de los fallecidos eran mujeres; mientras que en otros estudios ${ }^{(8,10,12,13,17)}$, la mortalidad más alta se presentó en los hombres mayores a 60 años.

Ante estos resultados, podemos señalar que, si bien las mujeres son menos propensas a requerir atención por el COVID-19 con respecto a los hombres, sí tienen mayor riesgo de fallecer, sobre todo el grupo mayor de 70 años; es por ello que consideramos adecuado catalogarlas como pacientes de alto riesgo. Sin embargo, creemos que estos resultados no serían exactos, ya que la población femenina de nuestro estudio es poco numerosa (14 mujeres), por lo cual se sugiere investigar más este aspecto. 
Características epidemiológicas de los pacientes atendidos por COVID-19 en el

Servicio de Emergencia del Hospital Militar Central Luis Arias Schreiber

Encontramos que 60 pacientes $(67,41 \%)$ tenían por lo menos una comorbilidad, y $29(32,59 \%)$ no tenían ninguna. Estos hallazgos coinciden con otros estudios ${ }^{(12-14)}$ cuya población presentó, en su mayoría, comorbilidades. Además, cabe resaltar que, tanto en investigaciones previas y en nuestro trabajo, la presencia de una comorbilidad aumenta el riesgo y mal pronóstico.

Se evidencia que las comorbilidades más frecuentes son la obesidad, sobre todo en el grupo etario de 50 a 59 años, seguida por la HTA y, en tercer lugar, por la DM2. Esto refleja una diferencia con las investigaciones que señalan a la HTA como la comorbilidad más frecuente ${ }^{(7-9,11-15,19)}$. Cabe resaltar que en nuestro estudio las comorbilidades se presentaron en el grupo etario de 40 años a más. Los cinco pacientes menores de 39 años no tuvieron ninguna. Esto plantea que el riesgo de necesitar asistencia médica aumenta en los mayores de 40 años debido a que es la edad a la que empezaron a presentar comorbilidades.

Los trabajos de Acosta $G$ et al. y otros autores muestran que los enfermos con COVID-19 presentaban una o más comorbilidades $(7,8,12,13,20)$. Según nuestros hallazgos, si aparece una sola comorbilidad, esta es la obesidad; si existen dos, la más frecuente es la hipertensión arterial; y por último, la obesidad y la HTA muestran la misma frecuencia si el paciente tiene tres comorbilidades. Por ello, reafirmamos que la obesidad y la HTA son las principales enfermedades que interferirían en la necesidad de atención continua por emergencia de los pacientes con COVID-19.

El $67,41 \%$ de los pacientes usó ventilación no invasiva (60 personas) y de ellos, 11 también requirieron ventilación mecánica. Este estudio mostró que la ventilación mecánica fue administrada solo a 19 pacientes $(21,34 \%)$, mientras que $70(78,66 \%)$ no la usaron. Llaro MK et al. y Villagran KA et al. reportan que la ventilación mecánica fue usada en más del $69 \%$ de los pacientes estudiados ${ }^{(11,17)}$. De acuerdo con lo hallado, la ventilación mecánica se empleó con muy poca frecuencia, por lo cual sugerimos realizar más estudios al respecto para definir la razón de este hecho.

Los resultados de este estudio se obtuvieron a partir de la información de los pacientes con COVID-19 atendidos y monitorizados en el Servicio de Emergencia del Hospital Militar Central en el periodo julio-octubre del 2020, por lo cual no son extrapolables a la población en general. Además, estos hallazgos se limitan a la evolución de los pacientes mientras permanecieron en el servicio estudiado, por lo que la evolución final podría haber variado.

Agradecimientos: A todo el personal de salud que lucha día a día contra esta pandemia con fortaleza, fe y esperanza; y al General de Brigada Herbet Dávila Ramírez por su apoyo incondicional.
Contribuciones de los autores: Katia Michelle Pezo Dianderas, Diego Rolando Chávez Fernández y Raúl Ernesto Porras Serna participaron en la recolección de datos, el diseño del estudio, la revisión de la literatura, el análisis e interpretación de resultados y la redacción del artículo.

Fuentes de financiamiento: El artículo ha sido financiado por los autores.

Conflictos de interés: Los autores declaran no tener ningún conflicto de interés.

\section{REFERENCIAS BIBLIOGRÁFICAS}

1. O'Brien N, Barboza M, Ventura J, Caycho T, Sandoval JS, López W, et al. Nuevo coronavirus (COVID-19). Un análisis bibliométrico. Rev Chil Anest. 2020; 49(3): 408-15.

2. Valdivia GG, Domínguez AD, Álvarez S, Meneses DM. COVID-19: fisiopatología y propuestas terapéuticas en investigación clínica. Rev Cent Investig Univ Salle. 2020; 14(53): 133-58.

3. Coronavirus Resource Center. Mapa COVID-19 [Internet]. Johns Hopkins University of Medicine; 2020. Disponible en: https:// coronavirus.jhu.edu/map.html

4. Organización Panamericana de la Salud. COVID-19 - Respuesta de la OPS/OMS Reporte 32 (2 de noviembre de 2020) - OPS/OMS I Organización Panamericana de la Salud [Internet]. OPS; 2020. Disponible en: https://www.paho.org/es/documentos/covid-19respuesta-opsoms-reporte-32-2-noviembre-2020

5. Ministerio de Salud. Situación actual COVID 19 Perú 2020 - 31 de octubre [Internet]. Lima; 2020. Disponible en: https://www.dge. gob.pe/portal/docs/tools/coronavirus/coronavirus311020.pdf

6. Serra MA, Las enfermedades crónicas no transmisibles y la pandemia por COVID-19. Rev Finlay. 2020; 10(2): 78-88.

7. Gamarra RR. Current Clinical-Epidemiological Profile of Patients with COVID-19 in Reference Hospital of Peru. J Biomed Res Environ Sci. 2020; 6(8): 81-5.

8. Acosta G, Escobar G, Bernaola G, Alfaro J, Taype W, Marcos C, et al. Caracterización de pacientes con COVID-19 grave atendidos en un hospital de referencia nacional del Perú. Rev Perú Med Exp Salud Pública. 2020; 37(2): 253-8.

9. Escobar G, Matta J, Taype W, Ayal R, Amado J. Características clínicoepidemiológicas de pacientes fallecidos por COVID-19 en un hospital nacional de Lima, Perú. Rev Fac Med Hum. 2020; 20(2): 14-9.

10. Giudice OE, Lucchesi EP, Belaúnde MT, Pinedo RH, Camere MA, Daly A, et al. Características clínicas y epidemiológicas de 25 casos de COVID-19 atendidos en la Clínica Delgado de Lima. Rev Soc Peru Med Interna. 2020; 33(1): 15-24.

11. Llaro MK, Gamarra BE, Campos KE. Características clínicoepidemiológicas y análisis de sobrevida en fallecidos por COVID-19 atendidos en establecimientos de la Red Sabogal-Callao 2020. Horiz Med. 2020; 20(2): e1229.

12. Cobas L, Mezquia N, Armenteros SS. Características clínicas de pacientes con sospecha de COVID-19 ingresados en el hospital "Frank País García”, La Habana. Rev Electrónica Dr Zoilo E Mar Vidaurreta. 2020; 45(4).

13. Biagi A, Rossi L, Malagoli A, Zanni A, Sticozzi C, Comastri G, et al. Clinical and epidemiological characteristics of 320 deceased patients with COVID-19 in an Italian Province: A retrospective observational study. J Med Virol. 2020; 92(11): 2718-24.

14. Urquiza Y, Pérez MD, Cortés A, Escalona I, Cabrales MO. Características clínico epidemiológicas de los pacientes de Las Tunas 
positivos al RT-PCR para la COVID-19. Rev Electrónica Dr Zoilo E Mar Vidaurreta. 2020; 45(4).

15. Tan SC. Clinical and epidemiological characteristics of Coronavirus Disease 2019 (COVID-19) patients. medRxiv. 2020.

16. Lechien JR, Chiesa CM, Place S, Laethem YV, Cabaraux P, Mat Q, et al. Clinical and epidemiological characteristics of 1420 European patients with mild-to-moderate coronavirus disease 2019. J Intern Med. 2020; 288(3): 335-44.

17. Villagran KA, Torrontegui LA, Entzana A. Características clínicoepidemiológicas de pacientes de COVID-19 en un Hospital de Sinaloa, México. REVMEDUAS. 2020; 10(2): 65-79.

18. Zhao J, Gao H-Y, Feng Z-Y, Wu Q-J. A Retrospective Analysis of the Clinical and Epidemiological Characteristics of COVID-19 Patients in Henan Provincial People's Hospital, Zhengzhou, China. Front Med. 2020; 7(286): 1-10.

19. Medina G, Carbajales EB, Figueredo Y, Carbajales Al, Silva I. Características clínico-epidemiológicas de pacientes positivos a la COVID-19 pertenecientes al policlínico “Joaquín de Agüero y Agüero", Camagüey. Rev Electrónica Dr Zoilo E Mar Vidaurreta. 2020; 45(4).

20. Aggarwal A, Shrivastava A, Kumar A, Ali A. Clinical and Epidemiological Features of SARS-CoV-2 Patients in SARI Ward of a Tertiary Care Centre in New Delhi. 2020. J Assoc Physicians India. 2020; 68(7): 19-26.
Correspondencia:

Katia Michelle Pezo Dianderas

Dirección: Av. San Felipe 647, dpto. 1904 - Jesús María, Lima, Perú.

Teléfono: +51981559818

Correo electrónico: 011300763h@uandina.edu.pe

Recibido: 30 de enero de 2021

Evaluado: 26 de marzo de 2021 Aprobado: 12 de abril de 2021

( La revista. Publicado por Universidad de San Martín de Porres, Perú. (c) ${ }_{\text {BY }}$ Licencia de Creative Commons Artículo en acceso abierto bajo términos de Licencia Creative Commons Atribución 4.0 Internacional. (http://creativecommons.org/licenses/by/4.0/)

ORCID iDs

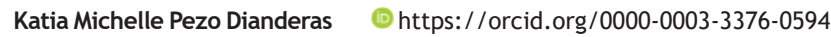
Diego Rolando Chávez Fernández @ https: / / orcid.org/0000-0001-7758-0099 Raúl Ernesto Porras Serna C https://orcid.org/0000-0003-1587-3587 